\title{
Resting-State Magnetoencephalography Reveals Neurobiological Bridges Between Pain and Cognitive Impairment
}

\author{
Yoshihito Shigihara (D) · Hideyuki Hoshi · Keisuke Fukasawa • \\ Sayuri Ichikawa · Momoko Kobayashi · Yuki Sakamoto • \\ Kazuyuki Negishi · Rika Haraguchi · Shin Konno
}

Received: September 15, 2020 / Accepted: October 15, 2020 / Published online: October 23, 2020

(C) The Author(s) 2020

\begin{abstract}
Introduction: Pain has been identified as a risk factor for cognitive dysfunction, which in turn affects pain perception. Although pain, cognitive dysfunction, and their interaction are clinically important, the neural mechanism connecting the two phenomena remains unclear.

Methods: The resting-state brain activity of 38 participants was measured using magnetoencephalography before and after the patients underwent selective nerve root block (SNRB) for the treatment of their pain. We then assessed the extent to which these data correlated with the subjective levels of pain experienced by the patients across SNRB based on the visual analogue scale and the cognitive status of the
\end{abstract}

Y. Shigihara $(\bowtie) \cdot$ H. Hoshi

Precision Medicine Centre, Hokuto Hospital, Obihiro, Japan

e-mail: y-shigihara@hokuto7.or.jp

Y. Shigihara · M. Kobayashi · Y. Sakamoto Precision Medicine Centre, Kumagaya General Hospital, Kumagaya, Japan

K. Fukasawa · S. Ichikawa · K. Negishi ·

R. Haraguchi

Clinical Laboratory, Kumagaya General Hospital,

Kumagaya, Japan

S. Konno

Department of Orthopaedics, Kumagaya General

Hospital, Kumagaya, Japan patients measured after SNRB using the Japanese versions of the Mini-Mental State Examination (MMSE-J).

Results: Slow oscillations (delta) in the right precentral gyrus, right middle temporal gyrus, and left superior frontal gyrus were negatively correlated with the subjective level of pain, and fast oscillations (gamma) in the right insular cortex and right middle temporal gyrus before SNRB were negatively correlated with the MMSE-J score afterwards. These correlations disappeared after SNRB.

Conclusion: The presently observed changes in neural activity, as indicated by oscillation changes, might represent the transient bridge between pain and cognitive dysfunction in patients with severe pain. Our findings underscore the importance of treating pain before a transient diminishment of cognitive function becomes persistent.

Keywords: Cognitive dysfunction; Dementia; Magnetoencephalography; Neural mechanism; Pain; Resting-state brain activities; Selective nerve root blocks; Spontaneous neural oscillations 


\section{Key Summary Points}

Why carry out this study?

Both pain and cognitive impairment are common symptoms that often coexist, especially in older individuals.

Although these two symptoms interact with each other, the neural mechanisms bridging them remain unclear.

The present study used the functional neuroimaging technique of magnetoencephalography to gain insight into the neural mechanisms.

\section{What was learned from the study?}

Resting-state brain activity recorded before and after the surgical treatment of severe pain correlated with subjective levels of pain and cognitive status when participants suffered from severe pain, but not after the pain was relieved.

The confirmed interaction between pain and cognitive function underscores the importance of treating pain to prevent patients from developing cognitive impairment.

\section{DIGITAL FEATURES}

This article is published with digital features, including a summary slide, to facilitate understanding of the article. To view digital features for this article go to https://doi.org/10.6084/ m9.figshare.13089305.

\section{INTRODUCTION}

Pain, like fever or fatigue, is an important bioalarm system that can be used to ensure the health and safety of the individual who experiences it $[1,2]$. It is a common, subjective, unpleasant sensory and emotional experience associated with actual or potential tissue damage. The lifetime incidence of radicular pain and low back pain ranges between 13 and $40 \%$ and between 50 and $80 \%$, respectively, with a higher prevalence in older populations [3-7]. Notably, pain is one of the major reasons for visits to medical facilities $[8,9]$.

As a multi-systemic phenomenon, pain relies on a widely distributed cortical/subcortical network known as the 'pain matrix' $[1,10]$ : a network that affects cognitive functions such as attention, memory, and executive function [11-18]. This link is evidenced by psychomotor slowing in patients with diabetic peripheral neuropathy [19], the association between the subjective level of pain and poor performance on executive functioning tasks in patients with rheumatoid arthritis [20], and the identification of pain as a risk factor for dementia [17]. On the other hand, cognitive status mediates the subjective level of pain. Patients with dementia exhibit larger responses to experimental nociceptive stimulation [21-23]. In clinical situations, patients with cognitive decline often complain of pain in response to low-level stimulation, such as the removal of surgical tape from their skin. However, while the interaction between pain and cognitive function is clear, the neural mechanisms underlying their relationship remain unclear [16].

Magnetoencephalography (MEG) is a noninvasive neuroimaging technique that is often used to record and evaluate brain activities in studies of pain and cognitive dysfunction [24-30]. Its widespread use is partly attributed to its ease, practicality, and safety of implementation; it requires only a few minutes to prepare, can complete a scan within $5 \mathrm{~min}$, and is easy to use repeatedly [31]. The present study compared the resting-state brain activity (spontaneous neural oscillations), subjective levels of pain, and cognitive status of patients before and after undergoing selective nerve root blocks (SNRB) to determine the relationship between pain and cognitive dysfunction at the neural level. 


\section{METHODS}

\section{Participants and Procedures}

Thirty-eight patients with low back pain were enrolled in this study (17 women; mean age, $68.9 \pm 10.3$ years; age range, $47-84$ years). They visited the outpatient Department of Orthopaedics at Kumagaya General Hospital, where they were diagnosed with sciatic pain by orthopaedic surgeons and were scheduled to receive SNRB for pain management. Patients reported pain on the left side $(n=22)$, right side $(n=11)$, both sides $(n=4)$, and midline $(n=1)$. This study was partly conducted during the period in which patients waited to receive surgery during ordinal clinical practice. Participants saw an orthopaedic surgeon who checked for any contraindications for receiving SNRB, and were provided explanations about the study and completed informed consent forms to indicate their agreement to participate. The first MEG scans and subjective pain assessments were performed in the MEG room (before $\mathrm{SNRB}$ ). The patients then received SNRB in the operating room. After the SNRB, the patients completed the second MEG scans and subjective assessments. Finally, each patient's cognitive status was assessed by clinical psychologists.

\section{Subjective and Cognitive Assessments}

The subjective level of pain of the participants was assessed using a visual analogue scale (VAS) before and after SNRB (pain-VAS). Pain-VAS scores range from 0 to 10 , where higher values indicated more severe pain. Cognitive status was assessed using the Japanese version of the Mini-Mental State Examination (MMSE-J) after the patients underwent SNRB [32, 33]. The MMSE-J score ranges from 0 to 30, where lower MMSE-J scores indicated more severe cognitive impairment. A cut-off score of 23/24 was used to indicate dementia [34]. We did not assess cognitive status before SNRB, in order to avoid the 'practice effect' (see Limitations). All procedures were completed within a few hours.

\section{MEG Scanning}

Spontaneous neural oscillations were recorded for 5 min using a 160-channel whole-head type magnetoencephalography system (RICOH1601: RICOH, Tokyo, Japan) before and after SNRB on the same day. During the scan, participants were placed in a magnetically shielded room and were asked to remain calm and to remain supine with their eyes closed. The scanning conditions were controlled for consistency, and the participants were made as comfortable as possible. The sensor and reference coils were gradiometers $15.5 \mathrm{~mm}$ in diameter and $50 \mathrm{~mm}$ at baseline, and each pair of sensor coils was separated by a distance of $23 \mathrm{~mm}$. The sampling frequency was $2000 \mathrm{~Hz}$. During the recording process, $500 \mathrm{~Hz}$ low-pass filtering was used. To co-register, the MEG source images with standard magnetic resonance imaging (MRI) structural brain images, and three fiducial magnetic marker coils were placed on each participant's face ( $5 \mathrm{~mm}$ above the nasion and bilaterally $10 \mathrm{~mm}$ in front of the tragus) during the MEG scan.

\section{MEG Analysis}

MEG data were preprocessed offline using the Statistical Parametric Mapping (SPM)-12 software package (Wellcome Trust Centre for Neuroimaging, London, UK; https://www.fil.ion. ucl.ac.uk/spm/) and the MEEG Automated Workflow (MEAW) system (https://www. hokuto7.or.jp/hospital/lang/english-home/ meaw/). The analytical procedure was performed as described previously [31]. Continuous MEG signals were divided into 10-s segments. Since a utility frequency was generated during the experimental environment, a $50-\mathrm{Hz}$ band-stop filter was applied to the epoched data. The filtered data were used directly for source-level analyses. To identify the specific locations of the brain regions responsible for producing the resting-state-induced components, source inversion procedures were applied separately to the oscillation components of delta $(0-3 \mathrm{~Hz})$, theta $(4-7 \mathrm{~Hz})$, alpha $(8-12 \mathrm{~Hz})$, beta $(13-25 \mathrm{~Hz})$, and gamma (low gamma, 
26-40 Hz; high gamma, 41-80 Hz). We used a maximal smoothness algorithm with a spatially coherent source model (i.e., $\mathrm{COH}$ algorithms implemented in SPM-12) [35], which is comparable to standardised low-resolution brain electromagnetic tomography (sLORETA) [36]. The $\mathrm{COH}$ algorithm is a popular source inversion algorithm that is often used in clinical environments [37, 38]. Using a single-shell model with canonical MR images provided by SPM-12, forward modelling was performed for the whole brain. The source inversion and estimation were performed by applying filters corresponding to each frequency band (from delta to high gamma). Source priors were not used for source estimation. The estimated oscillatory intensity at each location was saved as a 'source image' digital file in the Neuroimaging Informatics Technology Initiative (NIfTI) format. The source images were smoothed $(20 \times 20 \times 20 \mathrm{~mm})$ and used in the second (group)-level analysis. Two types of second (group)-level analyses were conducted (1) to determine whether the intensities correlated with subjective levels of pain (pain-VAS)

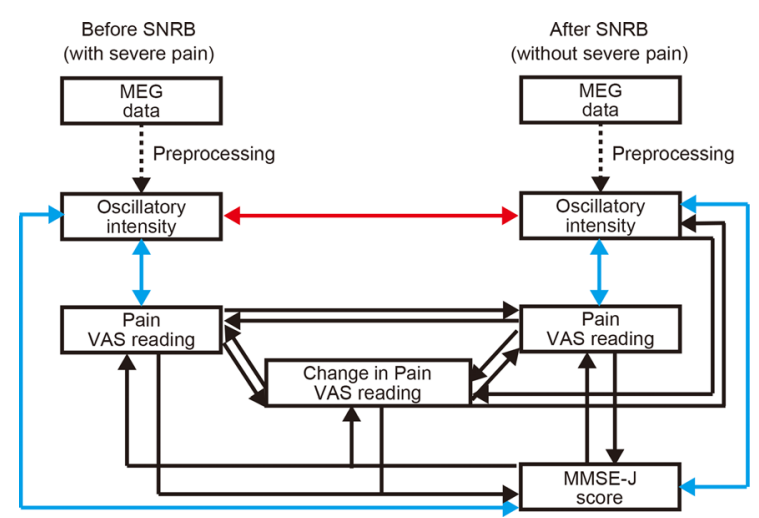

Fig. 1 Analytical procedure. The red arrow indicates analysis conducted to find brain regions where oscillatory intensities differed across SNRB. The blue arrows indicate analyses performed to find brain regions where oscillatory intensities correlated with the results obtained from subjective and cognitive assessments. The black arrows represent the comparison between the results collected from the subjective and cognitive assessments. Broken arrows represent MEG preprocessing. The participants' ages (a predictor used in the analysis procedure) is not described in this figure readings or cognitive status (MMSE-J score; blue arrows in Fig. 1), and (2) to find brain regions in which the intensities of the regional spontaneous neural oscillation differed across the SNRB (red arrow in Fig. 1). In the first analysis, the source images were separately regressed by the pain-VAS readings (before and after the SNRB) and MMSE-J scores. Both the positive and negative effects of the predictors were evaluated by building t-contrasts of +1 and -1 . In the second analysis, source images were compared for each patient using the paired $t$ test at each frequency band across SNRB. In this study, we report the source locations of peak level activations at a significance threshold of $P=0.05$ (corrected for family-wise error (FWE) rate) and a cluster extent at $\mathrm{k}>10\left(80 \mathrm{~mm}^{3}\right)$ [39]. Cortical regions where the peaks of the estimated sources were located were identified with SPM12.

\section{Statistical Analysis}

To evaluate the effects of the SNRB on the subjective experience of pain, we compared the pain-VAS readings across SNRB using a nonparametric bootstrapping approach. The changes in the score across SNRB were evaluated. The difference was resampled (with replacement) 20,000 times across all patients, and the percentage of the resampled differences, being larger or smaller than 0 (the smaller value), was taken as the significance level.

To evaluate the directional relationship between the subjective level of pain and cognitive status, we used a curve-fitting approach combined with non-parametric bootstrapping statistics. Pairs of predictor and response variables were prepared (Table 1). For each pair, predictor and response variables were resampled (with replacement) 20,000 times across all patients. In each iteration of the resampling, the response variable was fitted into one-dimensional (linear) and two-dimensional (quadratic) curves using a least-squares algorithm, and estimated coefficients were stored. Finally, the percentage of the resampled coefficients, being larger or smaller than 0 (the smaller value), was calculated for the linear and 
Table 1 Behavioural data of interest and results of polynomial fitting analysis

\begin{tabular}{|c|c|c|c|c|c|}
\hline \multirow[t]{2}{*}{ Predictor } & \multirow[t]{2}{*}{ Response } & \multicolumn{2}{|c|}{ Quadratic } & \multicolumn{2}{|l|}{ Linear } \\
\hline & & 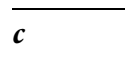 & $P$ & $c$ & $P$ \\
\hline \multirow[t]{3}{*}{ Pain-VAS before SNRB } & Change in pain-VAS & $<0.01$ & 0.394 & -0.50 & 0.221 \\
\hline & Pain-VAS after SNRB & -0.01 & 0.396 & 0.52 & 0.483 \\
\hline & MMSE-J & 0.09 & 0.119 & -1.15 & 0.137 \\
\hline Change in pain-VAS & MMSE-J & $0.07^{*}$ & 0.015 & $0.50^{*}$ & 0.042 \\
\hline Pain-VAS after SNRB & MMSE-J & 0.08 & 0.127 & -0.70 & 0.104 \\
\hline \multirow[t]{3}{*}{ MMSE-J } & Pain-VAS before SNRB & 0.01 & 0.363 & -0.47 & 0.367 \\
\hline & Change in pain-VAS & $<0.01$ & 0.466 & 0.08 & 0.427 \\
\hline & Pain-VAS after SNRB & 0.01 & 0.359 & -0.42 & 0.331 \\
\hline
\end{tabular}

Pain-VAS subjective level of pain measured by VAS, $S N R B$ selective nerve root block surgery, $c$ coefficient averaged across bootstrapping iterations, $P P$ values

*Indicates the coefficient was statistically significant (different from zero)

quadratic coefficients, respectively, which were then used as the significance level ( $P$ value). We report the average coefficients across the resampled data and the corresponding $P$ values.

\section{Compliance with Ethics Guidelines}

This study was conducted in accordance with the Declaration of Helsinki and was approved by the Ethics Committee of Kumagaya General Hospital (approval number 19). The details of the study (i.e., the objectives and procedure) were explained by the orthopaedic surgeon (coauthor S.K.). He also explained that participants could decline their participation at any time without stating their reasons, and that this would not lead to any disadvantage in terms of their clinical treatment. All participants gave written informed consent to participate in this study.

\section{RESULTS}

\section{Subjective and Cognitive Assessments}

The mean pain-VAS $( \pm$ SD) readings were $7.32 \pm 2.02$ before SNRB and $2.39 \pm 2.51$ after
SNRB. The change across SNRB was statistically significant $(P<0.001)$. Polynomial curve-fitting analysis revealed that the MMSE-J score was predicted by the change in pain-VAS readings (Table 1). Both quadratic (average: 0.07, $P=0.015$ ) and linear (average: $0.50, P=0.042$ ) coefficients were statistically larger than zero. No other directional relationships were found between the four factors: pain-VAS readings before and after SNRB, change in pain-VAS readings, and MMSE-J score.

\section{Regional Spontaneous Neural Oscillations}

Before SNRB, while the patients experienced severe pain, the delta intensity in the right precentral gyrus, right middle temporal gyrus, and left superior frontal gyrus correlated negatively with the pain-VAS readings (Fig. 2, Table 2). High gamma intensity in the right anterior insula cortex and right middle temporal gyrus negatively correlated with the MMSE-J score. These regions overlapped around the right precentral gyrus. After SNRB, when the severe pain diminished, no significant correlation was found between the intensity of regional spontaneous neural oscillations at any frequency and the pain-VAS readings or MMSE-J 


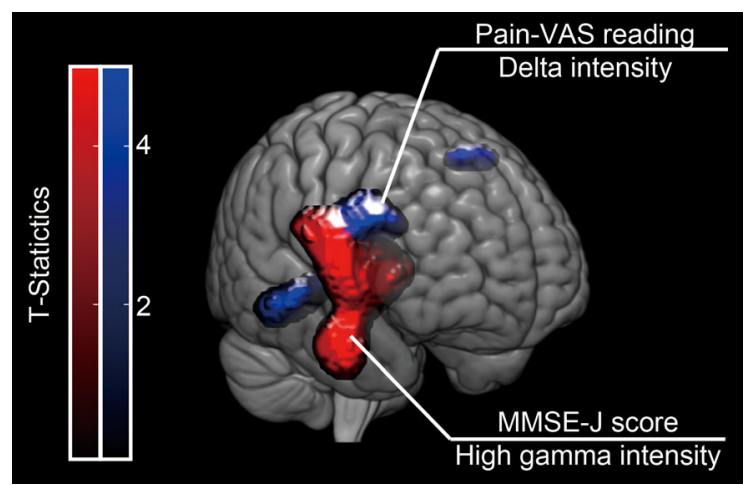

Fig. 2 Brain regions with oscillation intensities that correlated with subjective levels of pain and cognitive status. Blue colour indicates regions in which delta intensity was negatively correlated with the subjective level of pain (Pain-VAS). Red colour represents the region in which high gamma intensity was negatively correlated with cognitive status (MMSE-J score). The 3D image was created using MRIcroGL

scores. No significant changes in the intensity of the regional spontaneous neural oscillations were found at any frequency across SNRB.

\section{DISCUSSION}

The present study showed that regional spontaneous neural oscillations (i.e., resting-state brain activity) were correlated with subjective levels of pain (pain-VAS) and cognitive status (MMSE-J score) in participants suffering from severe pain (before the SNRB), but not after the severe pain was relieved (after the SNRB). More specifically, this study found that (1) the subjective level of pain correlated with regional spontaneous neural oscillations in the right temporal lobe at a low frequency when the pain was severe (before SNRB), (2) cognitive status was related to regional spontaneous neural oscillations in the right hemisphere at a high frequency when the pain was severe (before SNRB), and (3) these relationships disappeared after the severe pain was relieved (after SNRB).

Pain is an unpleasant, subjective experience from which approximately $40 \%$ of the general population suffers in one form or another $[40,41]$. Pain and cognitive dysfunction, including dementia, are particularly common among older individuals [3-6]; a new patient is diagnosed with dementia every 7 seconds [42].

Table 2 Correlation between resting-state oscillatory activity with subjective level of pain and cognitive status (corresponding to Fig. 1)

\begin{tabular}{|c|c|c|c|c|c|c|c|c|c|}
\hline & \multirow[t]{2}{*}{ Correlation } & \multicolumn{2}{|l|}{ Cluster level } & \multicolumn{2}{|l|}{ Peak level } & \multicolumn{3}{|c|}{ Peak coordinate } & \multirow[t]{2}{*}{ Brain region } \\
\hline & & $P($ FWE-corr $)$ & $\mathbf{k E}$ & $P($ FWE-corr $)$ & $T$ & $\bar{X}$ & $Y$ & $Z$ & \\
\hline \multicolumn{10}{|l|}{ Pain-VAS } \\
\hline \multirow[t]{3}{*}{ Delta } & \multirow[t]{3}{*}{ Negative } & 0.022 & 1883 & 0.003 & 5.185 & 54 & 12 & 26 & Rt precentral gyrus \\
\hline & & 0.025 & 1458 & 0.005 & 5.055 & 44 & -26 & -6 & $\begin{array}{l}\text { Rt middle temporal } \\
\text { gyrus }\end{array}$ \\
\hline & & 0.042 & 167 & 0.034 & 4.242 & -10 & 22 & 54 & $\begin{array}{l}\text { Lt superior frontal } \\
\text { gyrus }\end{array}$ \\
\hline \multicolumn{10}{|l|}{ MMSE-J } \\
\hline \multirow{2}{*}{$\begin{array}{l}\text { High } \\
\text { gamma }\end{array}$} & Negative & 0.015 & 6920 & 0.011 & 4.516 & 36 & 4 & 0 & Rt anterior insula \\
\hline & & & & 0.012 & 4.482 & 56 & 4 & -28 & $\begin{array}{l}\text { Rt middle temporal } \\
\text { gyrus }\end{array}$ \\
\hline
\end{tabular}

The $p$ values were corrected for multiple comparisons by the family-wise error (FWE) correction. $T, t$ value; $P, P$ value; $X$, $X$-coordinate; $Y, Y$-coordinate; $Z, Z$-coordinate. Pain-VAS, subjective level of pain measured by VAS 
These two conditions (pain and cognitive dysfunction) are demonstrably interrelated [11-14, 43], and pain has been identified as a risk factor for dementia [17]. However, the neural mechanisms underlying their interaction remain unclear.

The present study found that SNRB successfully reduced the subjective level of pain of the enrolled patients. The shift in the subjective levels of pain allowed us to investigate the correlation between pain and cognitive status. Subjective and cognitive assessments revealed a part of this bridge: SNRB was more effective [predictor] among patients (i.e., patients who experienced larger degrees of pain relief following SNRB) with higher MMSE-J scores after SNRB (i.e., better cognitive function) [response].

The oscillatory intensity analysis visualised regions whose activity correlated with subjective levels of pain and cognitive status under severe pain (before SNRB); these regions were found to overlap. Increased subjective levels of pain corresponded to the attenuated intensity of low-frequency oscillatory (delta) intensity in the right precentral gyrus, right middle temporal gyrus, and left superior frontal gyrus when the pain was very severe (i.e., before the SNRB; blue regions in Fig. 2). We ascribe the associations between oscillatory changes and the low frequency to the production of low-frequency spontaneous neural oscillations by the distributed networks, such as the pain system. We attribute the associations between oscillatory changes and the high frequency to the production of high-frequency spontaneous neural oscillations by local activity [44-51]. Studies have demonstrated that regional spontaneous neural oscillation at low frequency $(3-8 \mathrm{~Hz})$ is enhanced by chronic pain [52, 53]. Previous investigations have revealed that the 'pain matrix' consists of the primary and secondary somatosensory, insular, anterior cingulate, and prefrontal cortices and the thalamus [54]. The precentral gyrus is known as a primary motor area and contributes to the pain processing and chorionic pain reorganisation in the motor cortex; it is not, however, considered a component of the 'pain matrix' $[55,56]$. Hence, experiencing pain can affect regional spontaneous neural oscillations in the motor cortex.
The temporal gyrus also contributes to pain processing $[57,58]$. A previous study showed that painful stimulation evoked changes in local field potentials at low frequencies (theta) in the right medial temporal gyrus in humans [57]. Changes in low-frequency oscillations in the precentral and temporal gyri were also found in the right hemisphere. These findings are consistent with the observations that pain perception is dominated by the right hemisphere and that people are more sensitive to painful stimulation on the left side of the body [59-61]. Meanwhile, the left superior frontal gyrus also showed attenuated low-frequency oscillation accompanied by increasing levels of subjective pain. This finding could represent different changes in the precentral and middle temporal gyri because it is situated in the left hemisphere. The left superior frontal gyrus is known as a key component of the neural network of working memory $[62,63]$, although it contributes to pain processing as well [61]. Working memory deteriorates in the early stages of dementia [64], a trend that is consistent with the correlation between rostral lowfrequency oscillatory (delta) intensity and cognitive function $[65,66]$. Therefore, we speculate that attenuated low-frequency oscillatory (delta) intensity represents a part of the neural mechanism that bridges pain perception and cognitive dysfunction. Pain-related attenuation in the low-frequency oscillation (delta) affected cognitive regions, including the left superior frontal gyrus, across the 'pain matrix'.

The oscillatory intensity analysis also visualised brain regions whose activity was related to the patients' cognitive status under severe pain (i.e., before SNRB; red regions in Fig. 2). The MMSE-J score was negatively correlated with high-frequency oscillatory (gamma) intensity in the right insular cortex and right middle temporal gyrus, both of which contribute to pain perception. The right anterior insula is functionally connected with frontal regions and is considered to be a major component of the 'pain matrix' and the attention system $[15,54]$. The supposition that the right anterior insula modulates pain processing is supported by the observation that the experience of pain is affected by attention [67]. 
Atrophy of the right anterior insula in patients with Alzheimer's disease is related to their experience of hallucinations, which is a major symptom of dementia [68, 69]. Patients who experience hallucination achieve lower scores on the MMSE [69]. The previous observations agree with the present findings that high-frequency oscillatory intensity in the right anterior insula correlates with scores on the MMSE. The middle temporal gyrus also contributes to pain processing $[57,58]$, plays important roles in cognitive functions, and is affected by dementia [70-72]. A previous study showed that MMSE scores correlated with the volume of the right temporal gyrus [73]. It is reasonable that changes in oscillatory intensity in the right anterior insular cortex and the right middle temporal gyrus correlate with cognitive status, as assessed using the MMSE-J. However, it is unclear why cognitive dysfunction was associated with enhanced high-frequency oscillatory intensity in the rostral brain rather than enhanced low-frequency oscillatory activity in the caudal brain, which is often found in patients with dementia [30, 74-77]. The difference in frequencies (i.e., delta and high gamma) implies that different types of neural mechanisms underlie the neural activity in the regions [44-51], further suggesting that cognitive dysfunction-related oscillatory changes in the right insular cortex and right middle temporal gyrus are not the same as oscillatory changes in patients with 'genuine dementia'. The changes we observed should be considered pain-related transient changes because (1) brain regions affected by pain and cognitive dysfunction are close to each other and may even overlap (Fig. 2), (2) the oscillatory changes were only observed before SNRB (i.e., when the patients experienced severe pain), and (3) high- and lowfrequency oscillatory activity often interact with each other (cross-frequency coupling) [78]. High-frequency oscillatory activity represents regional activity, while lower-frequency oscillatory intensities are mainly produced by the distributed network [44-51]. High-frequency oscillatory activity in the right hemisphere is enhanced by the administration of non-pharmacological treatment to patients with dementia, which improves the patients' scores on the
MMSE-J [31]. We speculate that high-frequency oscillatory activity compensated for the paininduced cognitive dysfunction in these patients. The chief complaint of the participants was sciatic pain, and they did not notice their cognitive dysfunction in their daily lives. In other words, living their ordinary lives stimulated their brain and thus resembled a type of non-pharmacological treatment. Their engagement in daily activities enhanced highfrequency oscillatory intensity and effected a compensatory mechanism. The enhanced highfrequency oscillatory activity-i.e., the compensation-disappeared after SNRB, likely because the pain no longer disturbed the cognitive system. Although the high-frequency oscillatory activity could represent the preclinical state of dementia (i.e., transient cognitive dysfunction), it could lead to dementia due to cross-frequency coupling [78]. Therefore, in order to prevent clinical cognitive impairment, it is important to address any experienced pain.

At the group level, regional oscillatory intensities did not show significant changes across SNRB at any frequency in any participant (red arrow in Fig. 1). This finding reflects the fact that pain is processed by a widely distributed cortical/subcortical network (e.g. 'pain matrix') rather than a single brain region $[1,10]$. Individual differences in changes in regional oscillatory intensities would cancel out between participants.

The present study was subject to several limitations. First, pain is typically classified into binary categories of acute and chronic pain. We did not distinguish between them because it is difficult to determine whether sciatic pain is acute or chronic. Second, we did not divide the participants into subgroups based on the side of the body or type of body parts in which the pain was experienced. Although we used the participants' self-report to determine in which body parts they felt pain, most described the location ambiguously. The inaccuracy of the reported locations of pain could be due to 'non-specific, functional, and somatoform bodily complaints', which implies that pain is distributed across several locations, being a principle symptom of the patient's conditions $[79,80]$. Third, enhanced low-frequency oscillatory 
activity due to cognitive decline was not observed in the present study. We speculate that no participants suffered from severe cognitive decline, and the change was therefore not statistically significant. Fourth, cognitive status was assessed using MMSE-J only after SNRB. It is impractical to assess the same participant twice using the same neuropsychological assessment, since the participants always perform better on the second assessment (i.e., practice effect). In addition, from an ethical perspective, it is not appropriate to request that a patient in pain perform a task. Fifth, subjective and cognitive assessments did not show a direct relationship between the subjective level of pain and cognitive status either before or after SNRB. We ascribe this finding to selection bias. In the present study, we only enrolled patients who were scheduled to receive SNRB due to severe pain. Hence, patients with mild pain were not recruited (average and SD of pain-VAS readings were $7.32 \pm 2.02$ before the SNRB). Therefore, whether patients with mild or no cognitive impairment feel relatively less pain warrants further study in future research.

\section{CONCLUSIONS}

The activity of the pain system (i.e. 'pain matrix') was modulated by enhanced low-frequency oscillatory activity, and cognitive dysfunction under severe pain was related to enhanced high-frequency oscillatory activity. The regions in which these two oscillatory activities were observed overlapped in the right hemisphere; however, all oscillatory activity disappeared after the treatment of the pain with SNRB. It is plausible that these neural activities represent the transient bridge between pain and cognitive dysfunction. Therefore, our findings underscore the importance of pain before the transient bridge persists and aggravates cognitive dysfunction.

\section{ACKNOWLEDGEMENTS}

We thank the participants of this study and would like to affirm our genuine respect for their contributions to continued progress in the medical sciences.

Funding. This study was partially sponsored by RICOH (manufacturer of the magnetoencephalography system), which provided a joint research grant to Hokuto Hospital and Kumagaya General Hospital. Hokuto Hospital paid the Rapid Service Fee for the article using this funding award. The sponsor played no role in the study, its conception or design, the methods used, the analysis of the data, or the preparation of the paper.

Editorial Assistance. We would like to thank Yasmine Lashine from Editage (http:// www.editage.com) for providing English language editing services. Hokuto Hospital paid the English editing fee for the article using the funding award described in the Funding section.

Authorship. All named authors meet the International Committee of Medical Journal Editors (ICMJE) criteria for authorship for this article, take responsibility for the integrity of the work as a whole, and have provided their approval for this version of the manuscript to be published.

Disclosures. Yoshihito Shigihara leads the joint research projects that are supported by RICOH Co., Ltd. Hideyuki Hoshi is now employed by RICOH Co., Ltd. Keisuke Fukasawa, Sayuri Ichikawa, Momoko Kobayashi, Yuki Sakamoto, Kazuyuki Negishi, Rika Haraguchi, and Shin Konno have nothing to disclose. Rika Haraguchi is now Rika Sunaga, and is currently working at Shintoshin Musashino Clinic as a clinical laboratory technician.

Compliance with Ethics Guidelines. This study was conducted in accordance with the Declaration of Helsinki and was approved by the Ethics Committee of Kumagaya General Hospital (Approval number 19). The details of the study (i.e., the objectives and procedure) were explained by the orthopaedic surgeon (coauthor S.K.). He also explained that participants could decline their participation at any time 
without stating their reasons, and that this would not lead to any disadvantage in terms of their clinical treatment. All participants gave written informed consent to participate in this study.

Data Availability. Data are available from the Harvard Dataverse Data (Shigihara, Yoshihito, 2020, "Replication Data for: Pain and cognition", https://doi.org/10.7910/DVN/ 6BBFZX).

Open Access. This article is licensed under a Creative Commons Attribution-NonCommercial 4.0 International License, which permits any non-commercial use, sharing, adaptation, distribution and reproduction in any medium or format, as long as you give appropriate credit to the original author(s) and the source, provide a link to the Creative Commons licence, and indicate if changes were made. The images or other third party material in this article are included in the article's Creative Commons licence, unless indicated otherwise in a credit line to the material. If material is not included in the article's Creative Commons licence and your intended use is not permitted by statutory regulation or exceeds the permitted use, you will need to obtain permission directly from the copyright holder. To view a copy of this licence, visit http://creativecommons.org/licenses/by$\mathrm{nc} / 4.0 /$.

\section{REFERENCES}

1. Pia L, Garbarini F, Fossataro C, Fornia L, Berti A. Pain and body awareness: evidence from braindamaged patients with delusional body ownership. Front Hum Neurosci. 2013;7:298.

2. Watanabe $Y$, Kuratsune H. Brain science on chronic fatigue. JMAJ. 2006;49:19-26.

3. Stafford MA, Peng P, Hill DA. Sciatica: a review of history, epidemiology, pathogenesis, and the role of epidural steroid injection in management. Br J Anaesth. 2007;99:461-73.

4. Malec M, Shega JW. Pain management in the elderly. Med Clin North Am. 2015;99:337-50.
5. Meucci RD, Fassa AG, Faria NMX. Prevalence of chronic low back pain: systematic review. Rev Saude Publica. 2015;49:1.

6. Cao S, Fisher DW, Yu T, Dong H. The link between chronic pain and Alzheimer's disease. J Neuroinflammation. 2019;16:204.

7. Fatoye F, Gebrye T, Odeyemi I. Real-world incidence and prevalence of low back pain using routinely collected data. Rheumatol Int. 2019;39: 619-26.

8. Atlas SJ, Nardin RA. Evaluation and treatment of low back pain: an evidence-based approach to clinical care. Muscle Nerve. 2003;27:265-84.

9. Koes BW, van Tulder MW, Peul WC. Diagnosis and treatment of sciatica. BMJ. 2007;334:1313-7.

10. Iannetti GD, Mouraux A. From the neuromatrix to the pain matrix (and back). Exp Brain Res. 2010;205:1-12.

11. Dublin S, Walker RL, Gray SL, Hubbard RA, Anderson $\mathrm{ML}, \mathrm{Yu} \mathrm{O}$, et al. Prescription opioids and risk of dementia or cognitive decline: a prospective cohort study. J Am Geriatr Soc. 2015;63:1519-26.

12. van der Leeuw G, Eggermont LHP, Shi L, Milberg WP, Gross AL, Hausdorff JM, et al. Pain and cognitive function among older adults living in the community. J Gerontol A Biol Sci Med Sci. 2016;71: 398-405.

13. Scherder E, Herr K, Pickering G, Gibson S, Benedetti $\mathrm{F}$, Lautenbacher S. Pain in dementia. Pain. $2009 ; 145: 276-8$.

14. Whitlock EL, Diaz-Ramirez LG, Glymour MM, Boscardin WJ, Covinsky KE, Smith AK. Association between persistent pain and memory decline and dementia in a longitudinal cohort of elders. JAMA Intern Med. 2017;177:1146-53.

15. Wiech K, Lin C, Brodersen KH, Bingel U, Ploner M, Tracey I. Anterior insula integrates information about salience into perceptual decisions about pain. J Neurosci. 2010;30:16324-31.

16. Higgins DM, Martin AM, Baker DG, Vasterling JJ, Risbrough V. The relationship between chronic pain and neurocognitive function: a systematic review. Clin J Pain. 2018;34:262-75.

17. Ezzati A, Wang C, Katz MJ, Derby CA, Zammit AR, Zimmerman ME, et al. The temporal relationship between pain intensity and pain interference and incident dementia. Curr Alzheimer Res. 2018;16: 109-15. 
18. Jakobsson U, Klevsgård R, Westergren A, Hallberg IR. Old people in pain: a comparative study. J Pain Symptom Manag. 2003;26:625-36.

19. Ryan CM. Diabetes, aging, and cognitive decline. Neurobiol Aging. 2005;26:21-5.

20. Abeare CA, Cohen JL, Axelrod BN, Leisen JCC, Mosley-Williams A, Lumley MA. Pain, executive functioning, and affect in patients with rheumatoid arthritis. Clin J Pain. 2010;26:683-9.

21. Cole LJ, Farrell MJ, Duff EP, Barber JB, Egan GF, Gibson SJ. Pain sensitivity and fMRI pain-related brain activity in Alzheimer's disease. Brain. 2006;129:2957-65.

22. Kunz M, Mylius V, Scharmann S, Schepelman K, Lautenbacher S. Influence of dementia on multiple components of pain. Eur J Pain. 2009;13:317-25.

23. Lints-Martindale AC, Hadjistavropoulos T, Barber B, Gibson SJ. A psychophysical investigation of the facial action coding system as an index of pain variability among older adults with and without Alzheimer's disease. Pain Med. 2007;8:678-89.

24. Cheng Y, Yang CY, Lin CP, Lee PL, Decety J. The perception of pain in others suppresses somatosensory oscillations: a magnetoencephalography study. Neuroimage. 2008;40(4):1833-40.

25. Gopalakrishnan R, Burgess RC, Plow EB, Floden DP, Machado AG. A magnetoencephalography study of multi-modal processing of pain anticipation in primary sensory cortices. Neuroscience. 2015;304: 176-89.

26. Gross J, Schnitzler A, Timmermann L, Ploner M. Gamma oscillations in human primary somatosensory cortex reflect pain perception. PLoS Biol. 2007;5:1168-73.

27. Hari R, Baillet S, Barnes G, Burgess R, Forss N, Gross $\mathrm{J}$, et al. IFCN-endorsed practical guidelines for clinical magnetoencephalography (MEG). Clin Neurophysiol. 2018;8:1720-47.

28. Criado JR, Amo C, Quint P, Kurelowech L, Otis SM. Using magnetoencephalography to study patterns of brain magnetic activity in Alzheimer's disease. Am J Alzheimers Dis Other Dement. 2007;21: 416-23.

29. Fernández A, Maestú F, Amo C, Gil P, Fehr T, Wienbruch $\mathrm{C}$, et al. Focal temporoparietal slow activity in Alzheimer's disease revealed by magnetoencephalography. Biol Psychiatry. 2002;52: 764-70.

30. Fernández A, Turrero A, Zuluaga P, Gil-Gregorio P, Del Pozo F, Maestu F, et al. MEG delta mapping along the healthy aging-Alzheimer's disease continuum: diagnostic implications. J Alzheimer's Dis. 2013;35:495-507.

31. Shigihara Y, Hoshi H, Shinada K, Okada T, Kamada $H$. Non-pharmacological treatment changes brain activity in patients with dementia. Sci Rep. 2020;10:6744.

32. Folstein MF, Folstein SE, McHugh PR. "Mini-mental state". A practical method for grading the cognitive state of patients for the clinician. J Psychiatr Res. 1975;12:189-98.

33. Sugishita M, Hemmi I, Iwatsubo T. Japanese versions equivalent to original English neuropsychological tests in ADNI. Alzheimer's Dement. 2010;6: S348.

34. Mitchell AJ. The mini-mental state examination (MMSE): update on its diagnostic accuracy and clinical utility for cognitive disorders. In: Larner A, editor. Cognitive screening instruments. London: Springer; 2016. p. 37-48.

35. Friston K, Harrison L, Daunizeau J, Kiebel S, Phillips C, Trujillo-Barreto N, et al. Multiple sparse priors for the M/EEG inverse problem. Neuroimage. 2008;39: 1104-20.

36. Pascual-Marqui RD. Standardized low-resolution brain electromagnetic tomography (sLORETA): technical details. Methods Find Exp Clin Pharmacol. 2002;24(Suppl D):5-12.

37. Ray A, Bowyer S. Clinical applications of magnetoencephalography in epilepsy. Ann Indian Acad Neurol. 2010;13:14-22.

38. Terakawa Y, Tsuyuguchi N, Tanaka H, Shigihara Y, Sakamoto S, Takami T, et al. Quantitative analysis of MEG using modified sLORETA for clinical application. Clin Neurophysiol. 2008;119:1917-22.

39. Lieberman MD, Cunningham WA. Type I and Type II error concerns in fMRI research: re-balancing the scale. Soc Cogn Affect Neurosci. 2009;4:423-8.

40. Chung JWY, Wong TKS. Prevalence of pain in a community population. Pain Med. 2007;8:235-42.

41. Larsson C, Hansson EE, Sundquist K, Jakobsson U. Chronic pain in older adults: prevalence, incidence, and risk factors. Scand J Rheumatol. 2017;46: 317-25.

42. Ferri CP, Prince M, Brayne C, Brodaty H, Fratiglioni L, Ganguli M, et al. Global prevalence of dementia: a Delphi consensus study. Lancet. 2005;366: 2112-7. 
43. Cha DS, Carmona NE, Mansur RB, Lee Y, Park HJ, Rodrigues NB, et al. Pain and major depressive disorder: associations with cognitive impairment as measured by the THINC-integrated tool (THINC-it). Scand J Pain. 2017;15:62-7.

44. Bastos A, Userey M, Adams R, Mangun G, Fries P, Friston KJ. Canonical microcircuits for predictive coding. Neuron. 2012;76:695-711.

45. Buzsaki G, Bickford RG, Ponomareff G, Thal LJ, Mandel R, Gage FH. Nucleus basalis and thalamic control of neocortical activity in the freely moving rat. J Neurosci. 1988;8:4007-26.

46. Buzsáki G, Wang X-J. Mechanisms of gamma oscillations. Annu Rev Neurosci. 2012;35:203-25.

47. Colom LV. Septal networks: relevance to theta rhythm, epilepsy and Alzheimer's disease. J Neurochem. 2006;96:609-23.

48. Klimesch W. EEG alpha and theta oscillations reflect cognitive and memory performance: a review and analysis. Brain Res Brain Res Rev. 1999;29:169-95.

49. Rabiller G, He J-W, Nishijima Y, Wong A, Liu J. Perturbation of brain oscillations after ischemic stroke: a potential biomarker for post-stroke function and therapy. Int J Mol Sci. 2015;16:25605-40.

50. Sherman MA, Lee S, Law R, Haegens S, Thorn CA, Hämäläinen MS, et al. Neural mechanisms of transient neocortical beta rhythms: converging evidence from humans, computational modeling, monkeys, and mice. Proc Natl Acad Sci USA. 2016;113:E4885-94.

51. Steriade M. Corticothalamic resonance, states of vigilance and mentation. Neuroscience. 2000;101: 243-76.

52. Jeanmonod D, Magnin M, Morel A. Low-threshold calcium spike bursts in the human thalamus. Common physiopathology for sensory, motor and limbic positive symptoms. Brain. 1996;119:363-75.

53. Lenz FA, Kwan HC, Dostrovsky JO, Tasker RR. Characteristics of the bursting pattern of action potentials that occurs in the thalamus of patients with central pain. Brain Res. 1989;496:357-60.

54. Apkarian AV, Bushnell MC, Treede R-D, Zubieta J-K. Human brain mechanisms of pain perception and regulation in health and disease. Eur J Pain. 2005;9: 463-84.

55. Mercier C, Léonard G. Interactions between pain and the motor cortex: insights from research on phantom limb pain and complex regional pain syndrome. Physiother Can. 2011;63:305-14.
56. Xie Y-F, Huo F-Q, Tang J-S. Cerebral cortex modulation of pain. Acta Pharmacol Sin. 2009;30:31-41.

57. Liu CC, Ohara S, Franaszczuk P, Zagzoog N, Gallagher M, Lenz FA. Painful stimuli evoke potentials recorded from the medial temporal lobe in humans. Neuroscience. 2010;165:1402-11.

58. Moulton EA, Becerra L, Maleki N, Pendse G, Tully S, Hargreaves R, et al. Painful heat reveals hyperexcitability of the temporal pole in interictal and ictal migraine states. Cereb Cortex. 2011;21:435-48.

59. Merskey H, Watson GD. The lateralisation of pain. Pain. 1979;7:271-80.

60. Hari R, Portin K, Kettenmann B, Jousmäki V, Kobal G. Right-hemisphere preponderance of responses to painful $\mathrm{CO}_{2}$ stimulation of the human nasal mucosa. Pain. 1997;72:145-51.

61. Symonds LL, Gordon NS, Bixby JC, Mande MM. Right-lateralized pain processing in the human cortex: an FMRI study. J Neurophysiol. 2006;95: 3823-30.

62. Du BF, Levy R, Volle E, Seassau M, Duffau H, Kinkingnehun S, et al. Functions of the left superior frontal gyrus in humans: a lesion study. Brain. 2006;129:3315-28.

63. Yetkin FZ, Rosenberg RN, Weiner MF, Purdy PD, Cullum CM. FMRI of working memory in patients with mild cognitive impairment and probable Alzheimer's disease. Eur Radiol. 2006;16:193-206.

64. Baddeley A, Logie R, Bressi S, Della Sala S, Spinnler H. Dementia and working memory. QJ Exp Psychol A. $1986 ; 38: 603-18$.

65. Harmony T. The functional significance of delta oscillations in cognitive processing. Front Integr Neurosci. 2013;7:83.

66. Finnigan S, Robertson IH. Resting EEG theta power correlates with cognitive performance in healthy older adults. Psychophysiology. 2011;48:1083-7.

67. Chan SCC, Chan CCH, Kwan ASK, Ting K, Chui T. Orienting attention modulates pain perception: an ERP study. PLoS ONE. 2012;7:e40215.

68. Blanc F, Noblet V, Philippi N, Cretin B, Foucher J, Armspach JP, et al. Right anterior insula core region of hallucinations in cognitive neurodegenerative diseases. PLoS ONE. 2014;9:e114774.

69. Linszen MMJ, Lemstra AW, Dauwan M, Brouwer RM, Scheltens P, Sommer IEC. Understanding hallucinations in probable Alzheimer's disease: very low prevalence rates in a tertiary memory clinic. Alzheimers Dement (Amst). 2018;10:358-62. 
70. Cosentino S, Brickman AM, Griffith E, Habeck C, Cines S, Farrell M, et al. The right insula contributes to memory awareness in cognitively diverse older adults. Neuropsychologia. 2015;75:163-9.

71. Xie C, Bai F, Yu H, Shi Y, Yuan Y, Chen G, et al. Abnormal insula functional network is associated with episodic memory decline in amnestic mild cognitive impairment. Neuroimage. 2012;63:320-7.

72. Chan D, Anderson V, Pijnenburg Y, Whitwell J, Barnes J, Scahill R, et al. The clinical profile of right temporal lobe atrophy. Brain. 2009;132:1287-98.

73. Dinomais M, Celle S, Duval GT, Roche F, Henni S, Bartha R, et al. Anatomic correlation of the minimental state examination: a voxel-based morphometric study in older adults. PLOS ONE. 2016;11: e0162889.

74. Berendse HW, Verbunt JP, Scheltens P, van Dijk BW, Jonkman EJ. Magnetoencephalographic analysis of cortical activity in Alzheimer's disease: a pilot study. Clin Neurophysiol. 2000;111:604-12.

75. de Haan W, Stam CJ, Jones BF, Zuiderwijk IM, van Dijk BW, Scheltens P. Resting-state oscillatory brain dynamics in Alzheimer disease. J Clin Neurophysiol. 2008;25:187-93.

76. Jeong J. EEG dynamics in patients with Alzheimer's disease. Clin Neurophysiol. 2004;115:1490-505.

77. Osipova D, Ahveninen J, Jensen O, Ylikoski A, Pekkonen E. Altered generation of spontaneous oscillations in Alzheimer's disease. Neuroimage. 2005;27:835-41.

78. Roopun AK, Kramer MA, Carracedo LM, Kaiser M, Davies $\mathrm{CH}$, Traub RD, et al. Temporal interactions between cortical rhythms. Front Neurosci. 2008;2: 145-54.

79. McCracken LM, Faber SD, Janeck AS. Pain-related anxiety predicts non-specific physical complaints in persons with chronic pain. Behav Res Ther. 1998;36:621-30.

80. Schaefert R, Hausteiner-Wiehle C, Häuser W, Ronel J, Herrmann M, Henningsen P. Non-specific, functional, and somatoform bodily complaints. Dtsch Arztebl Int. 2012;109:803-13. 